\title{
Research on Gamma Ray Detections using Fiber Optic Radiation Sensors
}

\author{
P. Nivedha, R. Kumar, Dhanalakshmi Samiappan
}

\begin{abstract}
A comprehensive view of various techniques involved in detection of gamma rays, obtained from literatures has been presented. It also discusses with the current research activities carried out at various fronts, provides information about wide range of dosimeter available for detection of gamma rays. The dose range of each dosimeter is reviewed along with merits and demerits of various dosimeter techniques. Different types of sensing materials and their characteristics are discussed, with relative merits and demerits. The development of various types of optical fibres and their working principles are analyzed. The advantages of fibre Bragg grating over other fibres are also presented.
\end{abstract}

Keywords: Radiation Sensor, fiber optic radiation, dosimeter, gamma ray detection.

\section{INTRODUCTION}

The use of gamma ray has been increasing in our day to day life, particularly for medical, industrial and research applications. Hence the dosage detection of gamma rays is very essential as it decides the fatality limits. A number of physical instruments, devices with physical and chemical sensors are used for this purpose, but still researches are required to improve the performance, sensitivity, real time measurability, cost, etc. All these factors put together have to be taken into account for improved performance of the detector and dosimeter. This paper intends to provide the principles of various existing devices engaged in detection of gamma rays, with emphasis on the usage of optical fibers as signal carriers as well as sensors. The optical fiber sensors are emerging techniques in detection of Gama rays. There are different methods involved; the optical fiber which is directly made in contact with radiation source for detection purpose is said to be Intrinsic sensor, the optical fiber which is used as mediator in transmitting of optical signal is said to extrinsic sensor. Figure 1 shows the inner details and principles used in these methods of detection.

The Optical fibres act as a resistant to electrical and electromagnetic interference's, which are the major problems in the case of electrical dosimeter. Radiations induce electro motive forces which induces electric signal and in due electric noises that can cause damage the signal transmission or produce detection in the dosage [1]. The important property of optical fibres is that they can transmit the signal over long distances, without much distortion, at a greater speed when compared to electrical cables. There is

Revised Manuscript Received on September 10, 2019.

P. Nivedha, SRM Institute of Science and Technology, Department of Electronics and Communication Engineering,Chennai, Tamilnadu, India

R. Kumar, SRM Institute of Science and Technology, Department of Electronics and Communication Engineering,Chennai, Tamilnadu, India

Dhanalakshmi Samiappan, SRM Institute of Science and Technology, Department of Electronics and Communication Engineering,Chennai, Tamilnadu, India no need that the output unit which measures the dosage be located in same detecting area or in the place of sources which may be hazardous; thus, optical fibres help in remote operations. They can also be used in multiplexing process; i.e. several sensors can be joined together and monitor as a single output unit of sensing device. The recent development brought in radiation resistant fibres, which can withstand harsh environment where the radiation dosage is very high. This is particularly useful in vivo probes used in radiation therapy [2][3]. The dosage recommended generally for radiotherapy lies between 0 to $30 \mathrm{~Gy}$, but the linearity region lies only upto $5 \mathrm{~Gy}$, hence 0 to $5 \mathrm{~Gy}$ is considered to be suitable region for radiotherapy applications. Several advancements are made in radiation resistant fibres; the maximum tolerant range of the fibre is increased to 100KGy. The dopant concentration in the optical fibre materials can also be used to sense gamma radiation [4]. The length plays an important role in optical fibre detection. Optical fibre sensors show an increased sensitivity with the increase in length, which allows even lower doses to be measured [5], when length increases the attenuation increases, which leads to increase in sensitivity of the optical sensor. In recent years new technologies have been introduced by improving the durability of the fibre in the detection process which indicate even the level of corrosion can be assessed to sense the presence and dosage of the gamma rays. The introduction of scintillating material, either connected or mixed with optical fibres lead to new technology to detect and measure radiation [6].

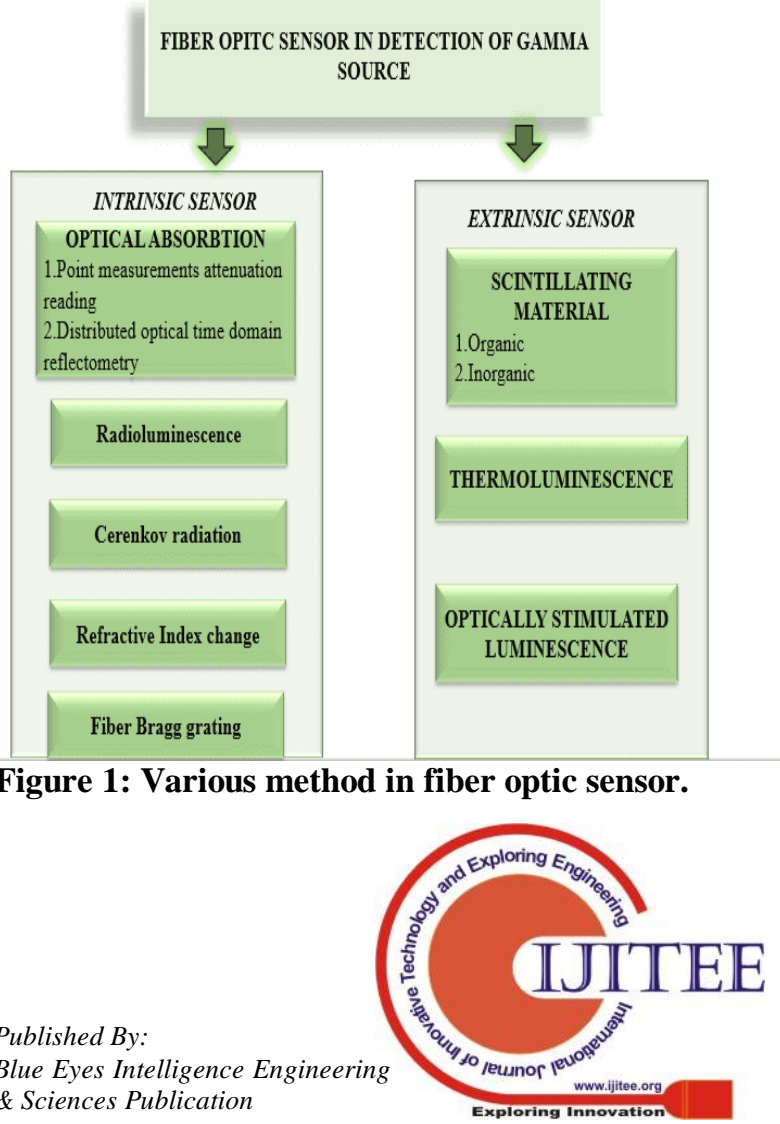




\section{CHARACTERISTIC AND PROPERTIES OF DOSIMETER}

Radiation dosimeter is designed based on the interaction between incident radiation and the sensor. This interaction of material with radioactive element acts as a sensor in detection of radiation dosage. In general, the types of interaction vary based on the types of dosimeter. Some produces electric charges in the presence of radiation. In optical sensor, scintillating material is used as sensing element. There should be minimum post irradiation for this occur due to the repair of the material caused during irradiation method. Immediately after irradiation, the dose count will be lost if the material begins to recover. This stability can be achieved with various range of treatment after the irradiation process, e.g. heat, strain [7]. Alternatively, do-pants material are added in order to avoid this post irradiation method. [8] [9]. In most of the optical system, the interrogated or reference wavelength is used in order to avoid post irradiation system. The real time dosimeter reduces the need for post irradiation system. Some material take average time of three to four hours responds with the radioactive elements to obtain accurate results [10], but for industrial uses the dosimeter needs to be designed in such a way that it provides the dosage instantaneously. The stability, durability, repeatability of the dosimeter also plays a very important role in the designing of dosimeter. In order to ensure these qualities, the instruments need to withstand several environmental conditions. Immunity towards harsh environment includes the operating temperature range and humidity range, in which the dosimeter can function within permitted accuracy [1]. For industrial use, the dosimeter must be designed in such a way that it is user friendly, even in harsh environment, without any risk of measurement. Finally, it is also important that it is available at low cost.

\section{INTRINSIC SENSOR}

\section{A. Optical absorption dosimeter}

Spectrophotometer is a device which is composed of a light source and a photometer. The light signal is allowed to get pass through the sample; the 80 absorbed light signal which gets emitted latter from the sample is allowed to pass through the photometer. The change in intensity of the light emitted is measured with the photo detector. This technique is used in optical absorption dosimeter. The first radiation dosimeter was invented in the year 1977 and used in navigation technology satellite. In this system several dopant materials are used in glass fibres for detection purpose. At earlier stage Zinc, Barium and Boron at low losses rate give linear response. One of the most common type of fibre is silica doped which has least sensitivity when compared to others.[5]. Also, these fibres are used effectively for neutron monitoring [7]. A new advancement in these types of sensors is phosphorous doped silica fibre [7][8][9]. The sensing method is based on Radiation Induced Attenuation (RIA) system. The advantage of this dosimeter is that they exhibit no tunneling effect and remain stable even after post irradiation [7]. Figure 2 show the characteristics. The results show the RIA changing linearly material also [7] for this purpose. The post irradiation fading

with dose, up to 220 Gy. As the dose rate increases, Sensitivity also increase along with the dose rate and thus suitable fiber are found out for dose rate between 1 and 100 $\mathrm{Gy} / \mathrm{h}$ [7]. In recent years, several advancements in optical fibres were made, which include plastic optical fibres, glass optical fibres, scintillating fibres, etc. The Ge doped optical fibres are used for ionizing radiations [11], it has a detection 100 limit of 360 MGy. Figure 3 indicate the saturation response starts at $10 \mathrm{~Gy}$. For Sio2 glass cladding scintillating fibre [14], the dose range lies between 1025.9 to 292.5 cGy. Figure 4 show the scintillating intensity of the fibre on interaction with the source and produce good linearity of R2 = 0. 9951 Plastic optic fibre are used in radiation detector nowadays.

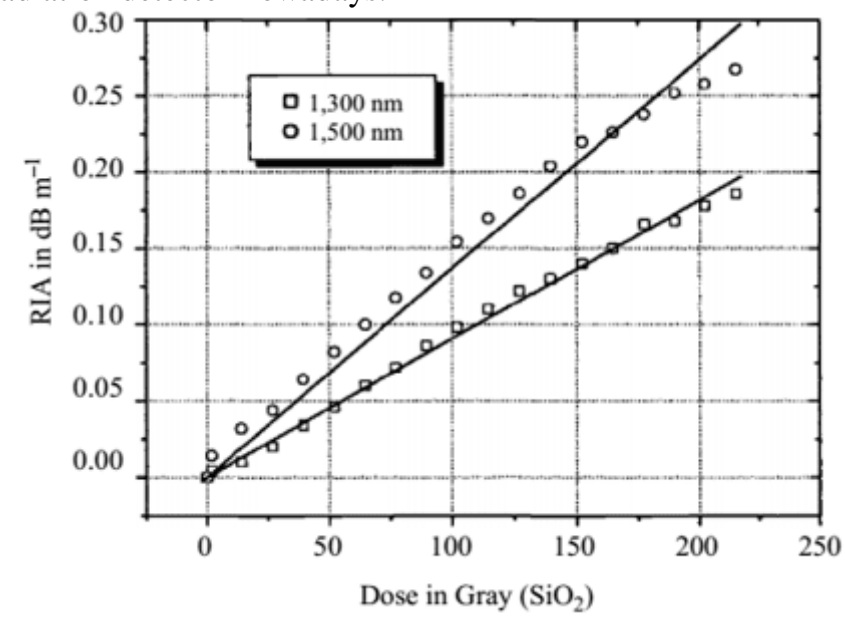

Figure 2: RIA Phosphorous doped optical fiber [7]

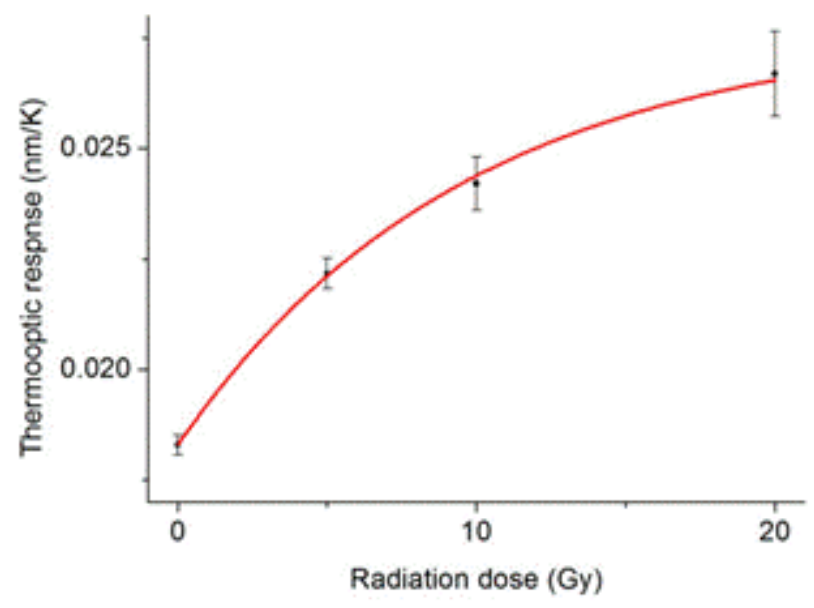

Figure 3: Thermo optic response of the Ge-doped optic fiber [11].

\section{B. Fiber Bragg grating $(F B G)$ method}

Fibre Bragg grating system is emerging technology in fibre optical sensors. It operates on wavelength shift properties. The shift in the wavelength of the signal gives the dosage value. This system operates on grating pitch and index of refraction of the material. More works are being carried out in the area of FBG, study is been carried on analyzing the effect of radiation on FBG and also the effect of FBG on dosage. The FBG sensors were initially

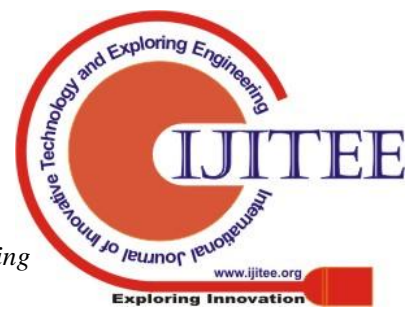


developed to suit harsh environmental conditions, where some of the radiations can be distracted measuredly, the remaining can be counted and the output will be presented cumulatively from the pre calibration, so as to adapt to high temperatures of nuclear environments [12][13][14][15][16]. The extension of this work, lead to the fabrication of FBG Sensor which works on Kramer Kronig dispersion relations [17]. The Ge doped FBG fibres [18] show a wavelength shift of $850 \mathrm{~nm}$ to $1,216 \mathrm{~nm}$. Figure 5 shows the shift in wavelength for different dose rate of gamma radiations. Figure 6 shows the Ge doped FBG optical fibre.

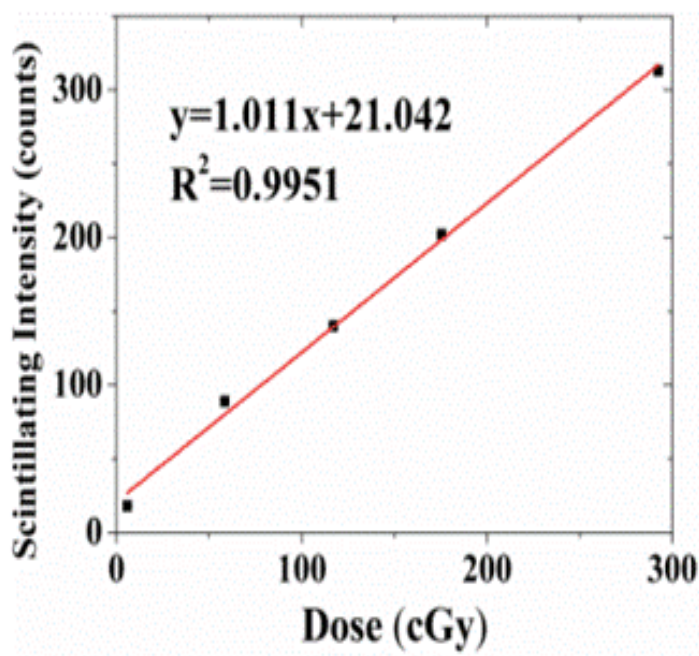

Figure 4: Scintillating intensity of Sio2 glass cladding fiber

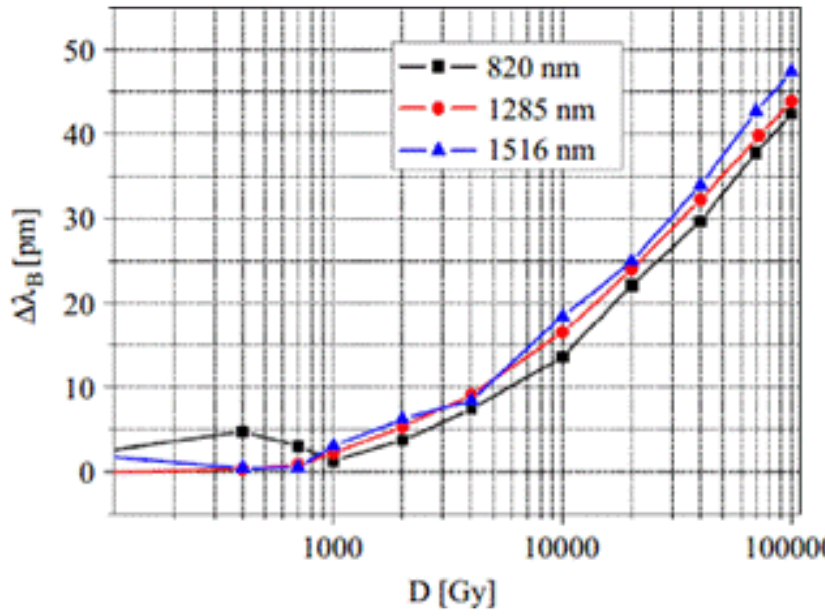

Figure 5: FBG on Gamma Radiation [18]

In spite of the fact that even a small change in temperature are considered for in sensor systems, the sensors generally require a highly sustainable structure, along with a constant steady temperature. The figure 7 explains about the radiation dosage, these sensors are used in the region where high sensitivity is needed within small range.

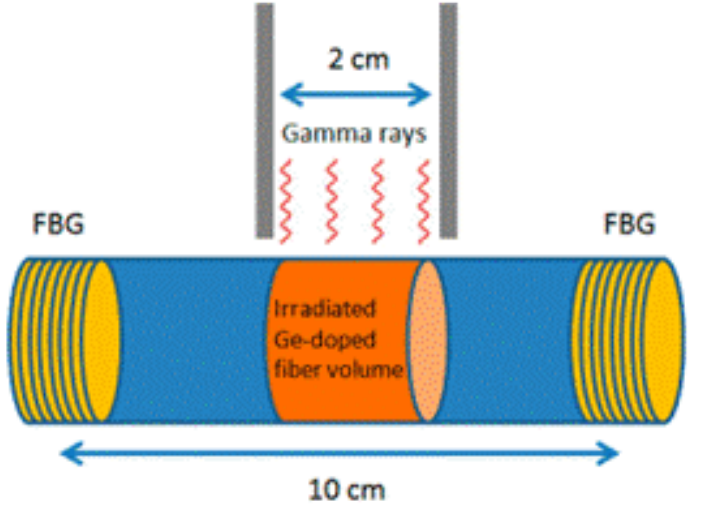

Figure 6: schematic diagram of Ge-doped Fiber Bragg Grating.[11].

Doses of the order of 1 Gy or less are typically involved in radiotherapy treatments. Still the research work is being carried out in order to improve the durability of the system and also the operating range.

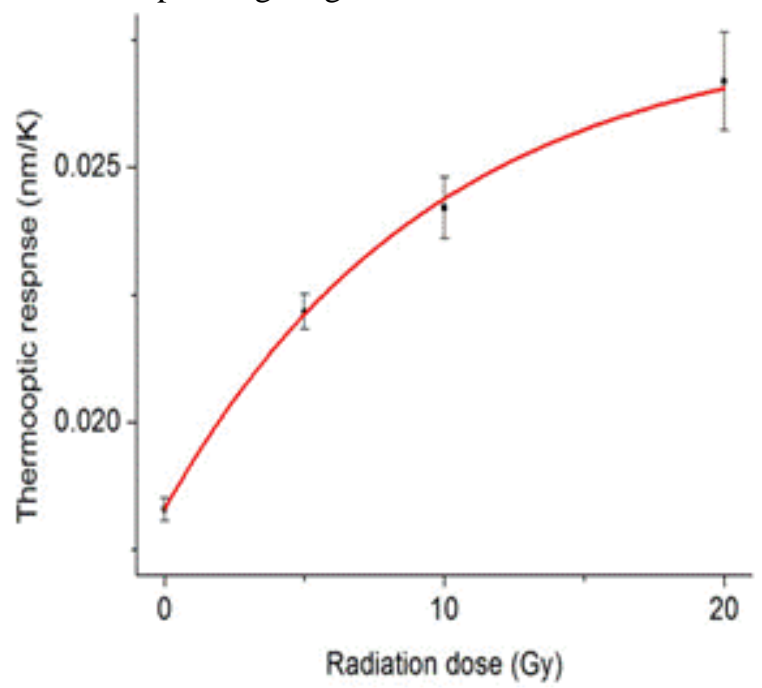

Figure 7: Gama dose rate.[11]

\section{EXTRINSIC SENSOR \& RESULTS}

\section{A. Scintillation Dosimeter}

Dosimeter on scintillation principle is one the oldest form of technique, in which the ionization radiation is converted into appropriate light signal with the help of emission of light by the scintillating material with which the radiation interacts. The light signal which get emitted from the detector is converted into electric signals with the photomultiplier tube or photodiodes. Scintillators can be subdivided into two set; inorganic scintillators and organic scintillators. Inorganic scintillators are generally in crystal form and are made up of alkali halides or oxides (e.g. CsI Sodium Iodide (NaI), Bismuth Germanate). Some crystal work intrinsically and some need external activator for scintillation. Thalium is one of the best example of this intrinsic crystal NaI(Tl) (Saint-Gobain Crystals, 2003). The Organic scintillator consist of aromatic hydrocarbons which is further subdivided into two; Liquid scintillator and Plastic 
scintillator. Sain global company has synthesised quit a number of scintillation crystals, some of them are NSL (tL), $\mathrm{Nal}(\mathrm{Tl}+\mathrm{Li}), \mathrm{LaBr} 3(\mathrm{Ce}), \mathrm{LaBr} 3(\mathrm{Ce}+\mathrm{Sr})$, LYSO etc. Each crystal has its own characteristic and working dosage ranges. This company is also working on gamma detection process for which they have also introduced a new fiber known as scintillating fibre (Saint-Gobain Crystals, accesable at: www.detectors.saint- gobain.com). Such Optical fibres are scintillating themselves to remote photomultiplier tube. A system proposed by [18] this technique is found to possess good linearity, robustness and small in size. The main drawback of the scintillation counters where the scintillating materials are attached with optical fibres is they can be employed to measure only low dosage radiations, in the range of $0.3-3,000 \mathrm{mGy} / \mathrm{h} 157$ [3][12][19]. Another type of sensor is developed by removing the cladding portion in small area and replacing with scintillation resin [20]. [21]. [22][19]. Fiber optic radiation with saphire based coating has been designed in where the luminescence light emitted from sapphire is measured. By using Beer Lamberts law, based on the thickness of plastic optic fiber the wavelength range and luminescence are measured. The figure 8 shows the performance of sapphire fiber optic sensor [23][6]

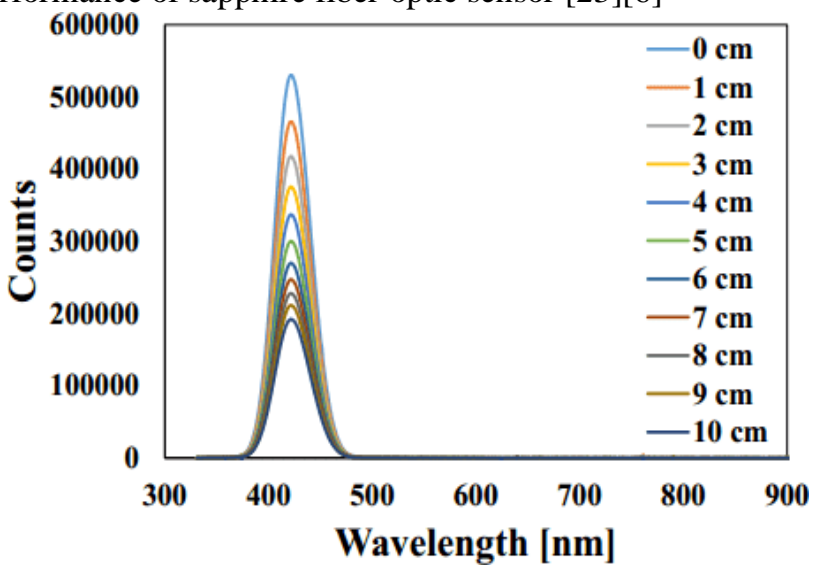

Figure 8: performance of sapphire based fiber optic sensor [23]

Another gamma ray spectrometer, designed with saint globin crystal, consists lutetium yttrium orthosilicate (LYSO:Ce) crystal of dimension $3 * 3 * 15 \mathrm{~mm}$ with $1 \mathrm{~m}$ long plastic optic fiber with diameter of $3 \mathrm{~mm}$ for detection of cesium 137 and cobalt 60.[23] [24]. The figure 9 given

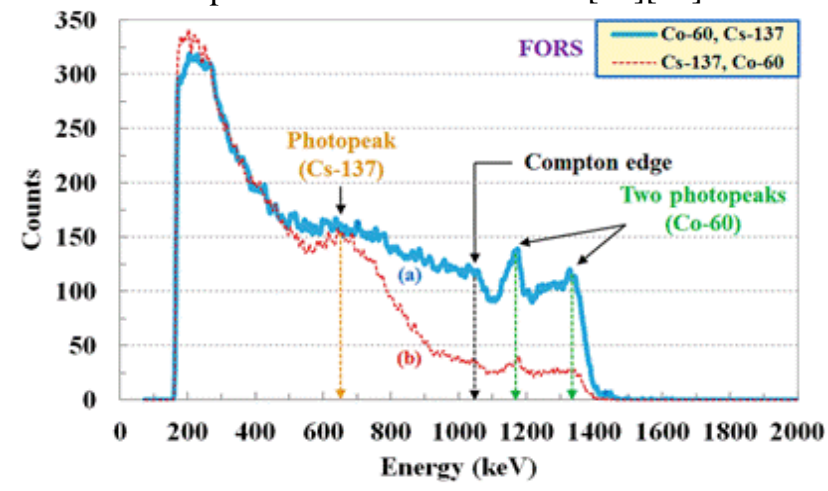

Figure 9: performance in detection of Cs 137 and Co 30 [24]. detection of gamma ray, emitted from Co-60 Source.[23][6], below show the performance of this sensor [23][24].

\section{B. Thermoluminescence Dosimeter (TLD)}

Thermo luminescence dosimeter is a type of dosimeter where light is emitted on getting heated. The major drawback of this type of dosimeter is it does not maintain a good linear response for large dosage range, it maintains a linear response up to few hundred grey. The commercially available thermo luminescence dosimeter is TLD-100 (LiF:Mg,Ti), where it can measure the dose rate up to to $1 \mathrm{Kgy}$ [19].This TLD dosimeter is more useful in medical field and environmental applications[22]. There is not much progress in development of thermoluminescence dosimeter, as it operates only at low range. there is not much progress in development of thermoluminescence dosimeter as it operates at low range.

\section{Optically Luminescence Dosimeter}

The optical luminescence dosimeter is similar to TLD, it also emits stored energy in the form of light after exposure with radiation. It is capable of operating at low dose range. The ability of the sensor is to calculate the extremely low dose range, since it is capable of measuring at low dose rate, it is used for personal dosimetry measurement and also in radiotherapy. This capability of measuring low dose rate has been used in wide range of application Eg: personal dosimeter in space for astronauts and in airport dosimeter measurement.[22]. They have wide range of application in medical field particularly in cancer treatment. A Linear Response of range around 0.01 to $10 \mathrm{~Gy}$ is obtained which is relevant to radiotherapy applications.

\section{CONCLUSION}

All the different types of optical fiber dosimeters were reviewed. Increasing number of project proposals, journal papers are coming up in the field of fiber optic radiation sensors. In general, the requirement for radiation dosimeter or sensors can be divided into two parts; they are for medical purpose and for environmental hazardless, as they are able to produce high sensitivity even at very low doses. The need for optical fiber dosimeter keeps on increasing because it has several advantages like interference of signals, transferability over a long distance, compatible size etc. The most widely used type of sensor in optical fiber dosimeter is extrinsic sensor. Research is still being carried out on improving the durability and quickness of the system. However, the system requires a well-constructed system which gives better efficiency, which seems improbable in industrial environments.

\section{REFERENCES}

1. T. Shikama, K. Toh, S. Nagata, B. Tsuchiya, Optical dosimetry for ionizing radiation fields by infrared radioluminescence, Measurement Science and Technology 17 (2006) 1103.

2. A. G. Mignani, S. Romano, F. Fusi, A. A. Mencaglia, Radiation dosimetry in radiotherapy: a model for an extrinsic optical fiber sensor, in: European Workshop on Optical Fibre Sensors, volume 3483, International Society for Optics and Photonics, pp. 99-103. 
3. D. A. Jackson, Novel fibre-optic-based ionization radiation probes, in: Second European Workshop on Optical Fibre Sensors, volume 5502, International Society for Optics and Photonics, pp. 234-239.

4. D. Alasia, A. F. Fernandez, B. Brichard, L. Abrardi, L. Th'evenaz, Study of the radiation effects on the properties of brillouin scattering in standard ge-doped optical fibres, in: 17th International Conference on Optical Fibre Sensors, volume 5855, International Society for Optics and Photonics, pp. 180-184. 221

5. A. Huston, B. Justus, P. Falkenstein, R. Miller, H. Ning, R. Altemus, Remote optical fiber dosimetry, Nuclear Instruments and Methods in Physics Research Section B: Beam Interactions with Materials and Atoms 184 (2001) $55-67$.

6. S. Novikov, A. Berintsev, V. Svetukhin, A. Alekseyev, A. Chertoriyskiy, R. Kuznetsov, V. Prikhodko, Simulating a scintillation fiber detector of the activities of ionizing radiation sources, Results in physics 6 (2016) 16-17.

7. B. Brichard, A. F. Fernandez, H. Ooms, P. Borgermans, F. Berghmans, True dose rate enhancement effect in phosphorous-doped fibre optic ra diation sensors, in: Second European Workshop on Optical Fibre Sensors, volume 5502, International Society for Optics and Photonics, pp. 184-188.

8. A. Kov'acs, M. Baranyai, P. Fuochi, M. Lavalle, U. Corda, S. Miller, M. Murphy, J. ODoherty, The application of sunna dosimeter film for process control at industrial gamma- and electron beam irradiation facilities, Radiation Physics and Chemistry 71 (2004) 329-333.

9. P. Borgermans, B. Brichard, F. Berghmans, F. Vos, M. Decreton, K. Golant, A. Thomashuk, L. Nikolin, On-line gamma dosimetry with phosphorous and germanium codoped optical fibres, in: Radiation and Its Effects on Components and Systems, 1999. RADECS 99. 1999 Fifth European Conference on, IEEE, pp. 477-482.

10. M. Watts, R. Bett, A new dosimetry system using a portable reader, Radiation Physics and Chemistry 63 (2002) 799-801.

11. S. Avino, V. D'Avino, A. Giorgini, R. Pacelli, R. Liuzzi, L. Cella, P. De Natale, G. Gagliardi, ionizing radiation detectors based on ge doped optical fibers inserted in resonant cavities, Sensors 15 (2015) 4242-4252.

12. A. F. Fernandez, B. Brichard, F. Berghmans, M. Decreton, Dose-rate dependencies in gamma-irradiated fiber bragg grating filters, IEEE Trans actions on Nuclear Science 49 (2002) 2874-2878.

13. A. Gusarov, F. Berghmans, O. Deparis, A. F. Fernandez, Y. Defosse, P. M'egret, M. Decreton, M. Blondel, High total dose radiation effects on temperature sensing fiber bragg gratings, IEEE Photonics technology letters 11 (1999) 1159-1161.

14. A. Gusarov, A. F. Fernandez, S. Vasiliev, O. Medvedkov, M. Blondel, F. Berghmans, Effect of gamma-neutron nuclear reactor radiation on the properties of bragg gratings written in photosensitive gedoped optical fiber, Nuclear Instruments and Methods in Physics Research Section B: Beam Interactions with Materials and Atoms 187 (2002) 79-86.

15. T. Kakuta, Development of new nuclear instrumentation based on optical sensing: irradiation effects on fiber bragg grating sensors, in: Four teenth International Conference on Optical Fiber Sensors, volume 4185, International Society for Optics and Photonics, p. 418547.

16. R. R. Maier, W. N. MacPherson, J. S. Barton, J. D. Jones, S. McCulloch, A. Fernandez- Fernandez, L. Zhang, X. Chen, Fiber bragg gratings of type $\mathrm{i}$ in smf-28 and b/ge fibre and type iia b/ge fibre under gamma radiation up to 0.54 mgy, in: 17 th International Conference on Op tical Fibre Sensors, volume 5855, International Society for Optics and Photonics, pp. 511515 .

17. K. Krebber, H. Henschel, U. Weinand, Fibre bragg gratings as high dose radiation sensors, Measurement Science and Technology 17 (2006) 2731095.

18. A. S. Beddar, S. Law, N. Suchowerska, T. R. Mackie, Plastic scintillation dosimetry: optimization of light collection efficiency, Physics in Medicine \& Biology 48 (2003) 1141.

19. S. O'Keeffe, C. Fitzpatrick, E. Lewis, A. Al-Shamma'a, A review of optical fibre radiation dosimeters, Sensor review 28 (2008) 136-142.

20. E. Takada, D. Yamada, H. Kuroda, A new optical fibre sensor for multipoint radiation measurement with sensing regions in its cladding, Measurement Science and Technology 15 (2004) 1479.

21. E. Takada, Y. Hosono, T. Kakuta, M. Yamazaki, H. Takahashi, M. Nakazawa, Application of red and near infrared emission from rare 12 earth ions for radiation measurements based on optical fibers, IEEE Transactions on Nuclear Science 45 (1998) 556-560.

22. B. Marczewska, P. Bilski, M. Budzanowski, P. Olko, V. Chernov, Dosimetry properties of tm-doped single caf2 crystals, Radiation measurements 33 (2001) 571-576.

23. Y. B. Song, K. W. Jang, H. J. Kim, D. E. Lee, M. Kim, S. H. Shin, 290 W. J. Yoo, B. Lee, Measurement of gamma-rays induced luminescence generated in a sapphire based fiber-optic radiation sensor, Journal of Applied Mathematics and Physics 4 (2016) 1503.

24. Y. B. Song, S. H. Shin, S. W. Song, H. J. Kim, S. Cho, B. Lee, Feasibility study on remote gamma spectroscopy system with fiber-optic radiation sensor, Journal of Radioanalytical and Nuclear Chemistry (2018) 1-6. 